\title{
Fighting every Crisis? \\ Zum Zusammenhang von Corona- und Klimakrise
}

\author{
Noab Marschner
}

\section{Einleitung}

Im Rahmen von Fridays for Future demonstrierten 2019 weltweit Millionen vor allem junge Menschen für entschiedenes politisches Handeln in der Klimakrise ${ }^{1}$. Mit den verheerenden Waldbränden in Australien und im Amazonas sowie dem zweiten Dürresommer in Folge in Deutschland stieg auch das öffentliche Interesse für die Folgen der Erderhitzung. Dann begann im Dezember 2019 die Coronapandemie. Die mediale und politische Aufmerksamkeit für die Klimakrise ist seitdem stark zurückgegangen. Vor allem zu Beginn der Pandemie waren Großdemonstrationen nur sehr eingeschränkt möglich und die Entwicklungen in der Coronakrise überschatteten die Sichtbarkeit anderer gesellschaftlicher Themen. Gleichzeitig bleibt die Klimakrise eines der drängendsten Probleme der Gegenwart. Auch in der Debatte um den Umgang mit der Pandemie kommt es zu Diskussionen, welche Beziehungen zwischen Klima- und Coronakrise existieren. Einerseits wird das Vorgehen gegen die Klimakrise als sekundär im Vergleich zur Coronakrise betrachtet, andererseits die Coronapandemie als Phase ökologischer Erholung gedeutet. Manche stufen den politischen Umgang mit der Pandemie als Beispiel ein, wie entschiedenes Handeln auch in der Klimakrise möglich wäre, wobei Unterschiede tendenziell in den Hintergrund geraten, oder deuten die Coronakrise als Möglichkeitsfenster für eine sozial-ökologische Transformation. In diesem Beitrag werden diese Diskurse einer kritischen Analyse unterzogen. Anleitend ist dabei die Fragestellung, auf welchen Grundannahmen die unterstellten $\mathrm{Zu}$ sammenhänge von Klima- und Coronakrise sowie die Maßnahmen gegen die Krisen aufbauen.

Um dieser Frage nachzugehen, ziehe ich zum einen Theorien heran, die sich mit der Diagnose des Anthropozäns beschäftigen, und zum anderen

1 Ich verwende vorwiegend den Begriff Klimakrise, um die enorme Geschwindigkeit des menschengemachten Klimawandels und dessen tiefgreifende Folgen für ökologische und soziale Prozesse zu benennen. 
solche, die globale und gesellschaftliche Machtverhältnisse in den Blick nehmen. Zunächst analysiere ich das Verständnis von Natur und Gesellschaft, das der Entgegensetzung von Klima- und Coronakrise zugrunde liegt. Danach gehe ich auf die Frage nach der Vergleichbarkeit beider Krisen hinsichtlich räumlicher und zeitlicher Dimensionen ein. Zuletzt kritisiere ich den dominanten Modus der Krisenbewältigung im Zuge der Coronakrise sowie dessen Einbettung in kapitalistische und nationalistische Strukturen, dem ich einen Appell für die integrative Behandlung multipler sozial-ökologischer Krisen gegenüberstelle.

\section{Coronakrise vs. Klimakrise? Der Natur-Gesellschaft-Dualismus}

In der Art, wie über den Zusammenhang von Corona- und Klimakrise gesprochen wird, fallen zwei Fehlschlüsse auf - von wirtschaftsliberaler und konservativer Seite einerseits, aus Teilen der Klimabewegung andererseits. Beide beruhen auf einem Dualismus von Natur und Gesellschaft als einander entgegengesetzte Sphären, der die wechselseitige Verwiesenheit sozialer und ökologischer Prozesse verkennt.

\section{Wirtschaft trumpft Klima}

Insbesondere konservative und wirtschaftsliberale Akteur*innen machten sich im Verlauf der Krise durch Äußerungen und Entscheidungen bemerkbar, die die aus der Pandemie resultierende Wirtschaftskrise als drängender bewerteten als die Klimakrise. In den Maßnahmen der Bundesregierung gegen die wirtschaftlichen Folgen der Pandemie zeigte sich die Dominanz dieses Ansatzes, für den die weitgehend konditionslose Rettung der Lufthansa und die Debatte um eine ,Neukaufprämie‘ für PKWs paradigmatisch sind. Das Ziel solcher Maßnahmen ist die Unterstützung von Industriezweigen, die massive Emissionen verursachen und für ein effektives Eindämmen des menschengemachten Klimawandels einer grundsätzlichen Transformation bedürften. In dem Maße, in dem klimapolitische Argumente einbezogen wurden, richteten sie sich auf technologischen Fortschritt, indem z.B. auf eine Erneuerung der Flotte oder eine Stärkung der individuellen Elektromobilität Bezug genommen wurde.

Ein solches Denken hat ein grundlegendes Problem: Es suggeriert eine Unabhängigkeit von Gesellschaft und Natur sowie eine Beherrschbarkeit ,der Natur ${ }^{6}$ durch Technologie. Kritisieren lässt sich dieses Narrativ mithil- 
fe von Theorien des Anthropozäns (vgl. Horn und Bergthaller 2019). Das Anthropozän bezeichnet als Gegenwartsdiagnose die „Einsicht, dass der Mensch tiefgreifend und im globalen Maßstab die Ökologie des Planeten verändert“ (ebd.: 9). So komme es zu einem „Bruch mit den ungewöhnlich stabilen ökologischen Verhältnissen des Holozäns“ (ebd.: 10), der vorangegangen erdzeitlichen Epoche, welche von geringen klimatischen Schwankungen geprägt war. Im Anschluss an Horn und Bergthaller verstehe ich das Anthropozän als explizit historisches Phänomen, dessen Ursachen nicht anthropologischer, sondern gesellschaftlicher und ökonomischer Art sind. Sie stehen in starker Verbindung mit dem Aufkommen des globalen Kapitalismus und den modernen Industriegesellschaften seit dem späten 18. Jahrhundert (vgl. ebd.: 35f.).

Bruno Latour (2017) bricht in seinen Überlegungen zum Anthropozän explizit die ontologische Trennung und Entgegensetzung von Natur und Gesellschaft auf und geht stattdessen von konstitutiven Wechselbeziehungen zwischen gesellschaftlichen und ökologischen Prozessen aus. Menschliche Gesellschaften beeinflussen demnach massiv die nicht-menschliche Umwelt, während zugleich ökologische Prozesse auf gesellschaftliche einwirken. Latour fasst Natur und Gesellschaft als gemeinsamen Nexus unter dem Begriff „Nature/Culture“ (ebd.: 16). Damit will er deren Trennung als Teil der westlichen Epistemologie und als Dualismus mit normativen Folgen offenlegen. Bezüglich des menschengemachten Klimawandels schlägt er vor, nicht von einer, ökologischen Krise‘ zu sprechen, mit der das Phänomen außerhalb der Gesellschaft verortet werde, sondern von einer „profound mutation in our relation to the world" (ebd.: 8).

Jason Moore (2017) ergänzt diese Diskussion, indem er den Begriff des Anthropozäns zum Kapitalozän abwandelt, und so gesellschaftliche Ausbeutungsverhältnisse fokussiert. Er situiert den Kapitalismus in einem „web of life“ (ebd.: 609), d.h. einer Welt-Ökologie, in der verschiedene Spezies miteinander interagieren. Der Gesellschaft-Natur-Dualismus behindere nicht nur die Analyse der Interaktion menschlichen und nicht-menschlichen Lebens, sondern reproduziere auch "systems of domination, exploitation and appropriation“ (ebd.: 601). Diese beschränkt Moore explizit nicht auf die Ausbeutung nicht-menschlicher Natur. Stattdessen bezieht er auch die Naturalisierung von Geschlechterverhältnissen und rassistischen Zuschreibungen mit ein, die gewaltsame Ausbeutungen von Menschen rechtfertigen.

Katharina Hoppe (2020) thematisiert ebenfalls die Herrschaftsfunktion von Dualismen am Beispiel von Natur und Gesellschaft. Im Anschluss an Donna Haraway führt sie aus, Dualismen seien mit gewaltsamen SubjektObjekt-Zuschreibungen verbunden, wobei die Gesellschaft als aktives Sub- 
jekt und die Natur als passives Objekt dargestellt werde. Das führe zur „Unfähigkeit, eine Eigenwilligkeit und Destruktivität des Natürlichen anzuerkennen, die sich einer kompletten Beherrschung entzieht“" (ebd.). So sei der Umgang mit dem zur ,Natur' Erklärten geprägt von Beherrschungsfantasien. Statt die Interaktion und Verwobenheit „bio- und ökosozialer Prozesse“ (ebd.) anzuerkennen, werde fälschlicherweise von der Möglichkeit der Beherrschung des Ökologischen durch das Soziale und Technologische ausgegangen.

Wird die Krisenbewältigung im Kontext der Coronapandemie vor allem als Handeln in einer Wirtschaftskrise betrachtet, verkennt das die konstitutive Verwobenheit ökologischer und gesellschaftlicher Prozesse. Zwar wird in einem gewissen Maße die Abhängigkeit ,der Gesellschaft' von ökologischen Grundlagen anerkannt - schließlich werden der menschengemachte Klimawandel und dessen Folgen nicht grundsätzlich geleugnet. Dennoch erscheint die Rettung der kapitalistischen Wirtschaftsordnung derart vorrangig, dass von Maßnahmen gegen die Klimakrise mindestens zeitweise abgesehen werden soll. Dadurch geraten die ökologischen Grundlagen auch der kapitalistischen Ökonomie weitgehend aus dem Blick. Besonders zum Vorschein tritt dies in der Argumentationsfigur, dass bei finanziellen Unterstützungsmaßnahmen auch an die Schulden gedacht werden müsse, die zukünftigen Generationen dadurch aufgebürdet würden. ${ }^{2}$ Dies ignoriert die ökologischen Folgen des Handelns, obwohl die Verantwortung gegenüber zukünftigen Generationen zugleich ein besonders prominentes Narrativ der Klimabewegung ist. Der Fokus auf ausschließlich technologische Lösungen zur Emissionsminderung u.a. in Flug- und Automobilbranche - anstelle etwa eines Wandels der Mobilität und der Formen des Wirtschaftens - lässt sich darüber hinaus als Beherrschungsfantasie einstufen, die von der Kontrollierbarkeit des Klimas durch technologischen Fortschritt ausgeht.

Gleichzeitig reproduziert diese Form der Krisenbewältigung eine Reihe von Unterdrückungsverhältnissen innerhalb von Gesellschaften, auf die im vierten Abschnitt näher eingegangen wird. Zuvor ist die Kehrseite des Natur-Gesellschaft-Dualismus Fokus der Analyse.

2 Exemplarisch ist das Zitat des FDP-Politikers Otto Fricke zum Konjunkturpaket der Bundesregierung vom Juni 2020: „Die Schuldenberge, die wir jetzt anhäufen, werden unsere Kinder und Enkel noch lange abtragen müssen“ (zitiert nach Lehmann 2020). 


\section{2. ,\#WirSindDasVirus}

Ein diskursiver Gegenpol zum wirtschaftlichen Modus der Krisenbewältigung ist die Debatte um ökologische Erholung durch die Coronakrise, welche besonders zu Beginn der Pandemie aufkam. Darin wird der Rückgang gesellschaftlicher Aktivität aufgrund der Krise, der u.a. eine kurzfristige Senkung der Treibhausgasemissionen bewirkte, als ein Segen für ,die Natur' dargestellt. Teils wird dabei die Metapher des Virus genutzt, um ökologische Zerstörung durch Menschen zu benennen. Statt die Coronapandemie als humanitäre Katastrophe zu sehen, erscheint das Virus als Notwehrreaktion ,der Natur', die sich gegen eine selbst zum Virus erklärte ,Menschheit' verteidigen müsse. Besonders in den sozialen Medien wurden solche Aussagen unter den Hashtags \#\#irSindDasVirus‘ und ,\#NatureIsHealing ${ }^{6}$ vorgebracht. ${ }^{3}$ Eine abgeschwächte Variante ließ sich auch innerhalb etablierter Medien finden, wobei die Möglichkeit der Erholung ,der Natur' durch die Maßnahmen zur Pandemiebewältigung hervorgehoben wurde. So fragte die Deutsche Welle in einem Video, ob die Pandemie gut für die Umwelt sei und beschrieb es mit den Worten: „Can the corona crisis help our planet to breathe again?" (Deutsche Welle News 2020).

Auf den ersten Blick erscheint der Diskurs um ökologische Erholung durch die Pandemie als Bruch mit dominanten Vorstellungen des Verhältnisses von Natur und Gesellschaft. Ökologische Prozesse gelten hier nicht als Ressourcen, die beliebig für wirtschaftlichen Wohlstand genutzt werden können. Dem Diskurs zugrunde liegt allerdings der gleiche Dualismus: Eine scheinbar homogene Menschheit wird dabei imaginiert als zerstörerische Kraft gegenüber einer positiv besetzten, ausschließlich nichtmenschlichen Natur. Einschränkungen des gesellschaftlichen Lebens aufgrund der Pandemie werden so zu Klima- und Naturschutzmaßnahmen umgedeutet und normativ positiv bewertet. In Form eines Anthropozentrismus mit negativem Vorzeichen bleibt der Natur-Gesellschaft-Dualismus also erhalten.

Die weiter oben ausgeführten Theorien behandeln menschliches und nicht-menschliches Wohlergehen sowie soziale und ökologische Krisen als verbundene Phänomene. Diese doppelte Kritik an gesellschaftlichen Verhältnissen kann als notwendige Bedingung angesehen werden, um Forde-

3 Zur Illustration eignet sich das Youtube-Video 6 Things that Prove That The Earth Is Healing mit über 700.000 Aufrufen (Stand Juni 2020). Die Verfassenden deuten als Zeichen für die ,Heilung der Erde‘ u.a. die Rückkehr von Wildtieren in Städte (Curly Tales 2020). 
rungen nach ökologischer Nachhaltigkeit als emanzipatorisch einzustufen. Das hier ausgeführte Narrativ lässt sich im Gegensatz dazu als zutiefst menschenfeindlich einordnen, denn es ignoriert menschliches Leid in Folge der Pandemie vollends. Zudem steht es neomalthusianischen Argumentationsfiguren nahe und macht gesellschaftliche Machtverhältnisse unsichtbar, wodurch es eine Kritik an diesen verhindert.

Der Neomalthusianismus geht zurück auf den britischen Ökonomen Thomas Robert Malthus, der 1798 in seinem Essay on the Principle of Population prophezeite, dass das stetige Bevölkerungswachstum innerhalb absehbarer Zeit die Nahrungsmittelproduktion überschreiten werde, was eine Beschränkung der Bevölkerungszahl erforderlich mache. Malthus’ Argumentation läuft darauf hinaus, diejenigen, die sich nicht selbst mit dem Überlebensnotwendigen versorgen können, dem Tod zu überlassen (vgl. Horn/Bergthaller 2019: 144). Er propagiert damit, wie auch spätere sozialdarwinistische Positionen, eine Vorstellung von Gesellschaft, wonach deren scheinbar ,fitteste، Mitglieder überleben, und leugnet strukturelle Gründe für Armut. Auch wenn im Diskurs um ökologische Erholung durch die Coronakrise nicht explizit der Tod bestimmter Menschen gefordert wird, so ist er doch geprägt von neomalthusianischen und sozialdarwinistischen Denkfiguren. Dass das Virus vor allem für vulnerable Bevölkerungsgruppen, wie Menschen mit Vorerkrankungen und Alte, tödlich endet, wird nicht thematisiert. Auch wird hingenommen, dass an Covid-19 in Deutschland überproportional viele migrantische Arbeiter*innen in Schlachthäusern erkrankten. Strukturelle Gründe dafür, warum z.B. Arbeiter*innen in prekären Verhältnissen besonders betroffen sind, werden ausgeblendet.

Dies deutet bereits den zweiten gefährlichen Aspekt des Diskurses an. Die monolithische Darstellung, der Menschheit' macht gesellschaftliche Unterdrückungsverhältnisse unsichtbar und verhindert so die Kritik an den politischen und gesellschaftlichen Auswirkungen beider Krisen. Es gerät aus dem Blick, dass Menschen im Globalen Süden überproportional von der Klimakrise betroffen sind, während die Verursachenden großenteils im Globalen Norden zu finden sind (vgl. Coventry/Okereke 2017: 366). Auch globale Ungleichheiten im Zugang zu Ressourcen zur Eindämmung des Virus und zur Anpassung an die Folgen der Klimakrise bleiben unsichtbar. Innergesellschaftliche Machtverhältnisse werden ebenso ausgeblendet. So leiden gerade Menschen in marginalisierten Positionen durch die Pandemie und die zur Abwehr ergriffenen Maßnahmen und werden ihrer Lebensgrundlage beraubt. Das gilt z.B. für Menschen in prekären Beschäftigungsverhältnissen und solche ohne Zugang zu gut ausgestatteten Gesundheitssystemen. 
Da das Narrativ der ökologischen Erholung durch die Coronakrise all diese Machtverhältnisse ausblendet, menschliches Leid ignoriert und sozialdarwinistische Züge trägt, ist es als grundlegend anti-emanzipatorisch einzustufen. Der zugrundeliegende Natur-Gesellschaft-Dualismus verhindert die Analyse der Verbindung verschiedener Krisen. Gefordert wird eine ,Rettung der Erde' vor dem Menschen statt eine Rettung der aktuellen Bedingungen auf dem Planeten für die Menschen und andere Lebewesen.

\section{Coronakrise $=$ Klimakrise $?$ Die Frage der Betroffenheit}

Ein weiterer Diskurs verknüpft die Klima- und die Coronakrise miteinander. Darin wird die Pandemie als Vorbild dafür begriffen, wie entschiedenes politisches Handeln auch bezüglich der Klimakrise möglich sei. Auch wenn die Diagnose zutrifft, dass die Coronapandemie die grundsätzliche Möglichkeit staatlicher Eingriffe in gesellschaftliche und wirtschaftliche Prozesse aufzeigt, greifen diese zu kurz und reproduzieren dabei gesellschaftliche Unterdrückungsverhältnisse (vgl. dazu Kap. IV). Zudem geraten bei der Gleichsetzung der beiden Krisen Unterschiede zwischen ihnen aus dem Blick. So werden die verschiedenen Betroffenheiten durch die beiden Krisen hinsichtlich räumlicher und zeitlicher Dimensionen ignoriert.

\section{Räumliche Dimension}

Auf den ersten Blick scheinen Corona- und Klimakrise eine grundlegende Gemeinsamkeit zu haben: Als globale Krisenphänomene beeinflussen sie Gesellschaften weltweit und verändern den Alltag nahezu aller Menschen. In beiden Krisen unterscheiden sich dabei der Grad und die Form der Betroffenheit enorm entlang verschiedener Differenzkategorien. Bei genauerer Betrachtung gibt es jedoch wichtige Unterschiede zwischen Klima- und Coronakrise - einerseits betrifft das die räumliche Verteilung der Krisen, andererseits die Ebenen, auf denen sich ihre Auswirkungen zeigen.

Hilfreiche Bezugspunkte in der Analyse der Unterschiede sind Überlegungen zu sogenannten Skaleneffekten, d.h. qualitativen Sprüngen, die auftreten, wenn man den Maßstab verändert, der bei der Beobachtung eines Phänomens angewendet wird (vgl. Horn/Bergthaller 2019: 180f.). Räumlich lassen sich die Ursachen und Folgen der Klimakrise als Skaleneffekt einstufen. Erst eine bestimmte Menge von Treibhausgasemissionen, weit 
jenseits jener individueller Akteur*innen, löst qualitative Veränderungen im Weltklima aus. Während aber politische Handlungsfähigkeit gesellschaftlich ausdifferenziert ist, werden die Auswirkungen der Erderhitzung erst bei der Betrachtung des planetaren Maßstabs sichtbar. Dieser ist wiederum für das Treffen von Entscheidungen im gegenwärtigen politischen Alltag weitgehend ungeeignet und unterminiert deshalb politisches Handeln (vgl. Clark 2012: 148f.).

Anna Tsing (2019) analysiert ökologische Krisen mithilfe des Begriffs nonscalability. Sie bezeichnet solche Projekte als skalierbar, die ohne grundlegende Veränderung expandieren können und auf andere Kontexte übertragbar sind. Diesen stellt sie nicht-skalierbare Prozesse gegenüber, die geprägt sind von einer grundsätzlichen Relationalität und Unvorhersehbarkeit, welche Expansion verhindert. Das kapitalistische Wirtschaftssystem baut auf skalierbare Elemente auf, die allerdings auf nicht-skalierbare soziale und ökologische Prozesse angewiesen und mit diesen verwoben sind (vgl. ebd.). Skaleneffekte können im Anschluss an Tsing als Auswirkung der Interaktion skalierbarer und nicht-skalierbarer Elemente eingestuft werden. Bei der Expansion kapitalistischer Produktionsverhältnisse zeigen sich dabei die Folgen der Interaktion der Ökonomie mit nicht-skalierbaren ökologischen Prozessen z.B. anhand der Klimakrise.

Diese Überlegungen sind auf die Vergleichbarkeit von Klima- und Coronakrise anwendbar. Zunächst handelt es sich bei Viren und bei Klimaveränderungen um nicht-skalierbare Elemente, die in Beziehung zu menschlichem Leben existieren und in unterschiedlichen Kontexten verschiedene Folgen haben. Corona- und Klimakrise zeigen deshalb die Verwobenheit menschlicher Gesellschaftsordnungen mit solchen nicht-skalierbaren, nicht-menschlichen Elementen auf. Allerdings treten die Folgen des Coronavirus einerseits und des menschengemachten Klimawandels andererseits auf unterschiedlichen Ebenen hervor. Das Coronavirus wird auf der Ebene einzelner Individuen durch Erkrankung sichtbar, die Folgen von Treibhausgasemissionen erst auf der Ebene des Planeten. Die Ansteckung bei Covid-19 findet über direkten Kontakt mit dem Virus statt und kann daher vielfach von Mensch zu Mensch nachverfolgt werden. Demgegenüber bleiben die Ursachen und Folgen der Erderhitzung wenig klar zuordenbar. So nehmen u.a. tropische Stürme durch Treibhausgasemissionen $\mathrm{zu}$, doch der einzelne Sturm ist nicht im Ausstoß eines bestimmten $\mathrm{CO}_{2}$-Moleküls begründet, was die Sichtbarkeit der Klimakrise reduziert. Zugleich macht sich die Klimakrise global differenziert bemerkbar und ist nicht auf den Emissionsausstoß in der entsprechenden Region zurückzuführen. Statt des Maßstabs menschlicher Gesellschaften muss der planetare Maßstab angewendet werden, um die Klimakrise erfassen zu können. Wie 
oben ausgeführt, verringert dies politische Handlungsfähigkeit in einem weitgehend nationalstaatlich organisierten System.

Globale Machtverhältnisse prägen zusätzlich die Reaktion auf Coronaund Klimakrise. So breitete sich das Virus entlang globaler Lieferketten und Reisewege im Frühjahr 2020 frühzeitig im Globalen Norden aus. Anders verhält es sich bei der Klimakrise, deren Auswirkungen sich zurzeit überproportional im Globalen Süden zeigen, so im Fall von Dürren in Ostafrika oder in Küstenregionen in Südostasien, die vom steigenden Meeresspiegel bedroht sind (vgl. IPCC 2014). Viele der betroffenen Staaten haben dabei marginale Positionen im globalen Machtgefüge. Auch das führt zu einem weniger entschiedenen Vorgehen in der Klimakrise.

\section{Zeitliche Dimension}

Zudem laufen Corona- und Klimakrise auf verschiedenen Zeithorizonten ab, wobei die Coronakrise im Vergleich zum menschengemachten Klimawandel als kurzfristiger Schock wirkt. Vom erstmaligen Auftreten des Virus Ende 2019 bis zu seiner globalen Ausbreitung vergingen nur wenige Monate; die Inkubationszeit von Covid-19 beträgt nur bis zu zwei Wochen. Im Gegensatz dazu beruht der menschengemachte Klimawandel auf der Freisetzung von Energie, die sich über Millionen von Jahren in Form fossiler Energieträger abgelagert hat. Als u.a. $\mathrm{CO}_{2}$ verbleibt der Großteil der Energie über Jahrhunderte innerhalb der Atmosphäre, die Folgen der Klimaveränderungen werden mehrere hunderttausend Jahre spürbar sein (vgl. Archer 2009: 11). Damit stellt sich die Beschränkung der Klimakrise auch auf zeitlicher Ebene als ein Problem der Kollision unterschiedlicher Skalen dar. Politisches und gesellschaftliches Handeln, das sich im Zeitraum weniger Jahre bis Jahrzehnte bewegt, wird konfrontiert mit der geologischen Zeitrechnung planetarer ökologischer Prozesse (vgl. Chakrabarty 2016: 23ff.). Die Betroffenen von der Klimakrise sind deshalb auch (aber nicht nur) zukünftige Generationen, deren Interessen beispielsweise in repräsentativen Demokratien nicht vertreten werden. Im Unterschied dazu sind die Betroffenen der Coronapandemie vor allem gegenwärtig lebende Menschen, die tendenziell eher die Möglichkeit haben, für ihre Forderungen einzutreten.

Zudem lässt sich die Coronakrise - in ihrer spezifischen Ausprägung als globaler gesundheitlicher Notfall - zumindest teilweise als vorübergehend einstufen. Die Aussicht auf einen Impfstoff gilt als absehbares Ende der Pandemie, auch wenn ihre politischen und gesellschaftlichen Folgen langfristig wirken werden. Die Eingebundenheit menschlicher Gesellschaften 
in ökologische Prozesse und ihre generelle Anfälligkeit für Krankheitserreger zeigt sich ebenfalls als dauerhaft potenzielle Krise am Coronavirus. Die Krankheit selbst bleibt jedoch voraussichtlich zeitlich begrenzt. Die Klimakrise bedeutet hingegen das Ende einer geologischen Epoche und den Eintritt in eine neue. Sie lässt sich treffender als eine Reihe zunehmender Krisen beschreiben, die u.a. in Dürresommern, Waldbränden und Extremwetterereignissen sichtbar werden. Das meint Bruno Latour, wenn er die Klimakrise nicht als ökologische Krise, sondern als Mutation unserer Beziehung zur Welt beschreibt (vgl. Latour 2017: 8). Horn und Bergthaller kategorisieren das Anthropozän in diesem Zusammenhang als „unbemerktes, aber irreversibles Überschreiten einer Schwelle“" (Horn/Bergthaller 2019: 203).

Eine Gleichsetzung von Klima- und Coronakrise gestaltet sich mit Rückbezug auf die unterschiedlichen räumlichen und zeitlichen Wirkungsmechanismen als problematisch. Daher ist es vorschnell, Regierungshandeln in der Coronakrise als Beispiel dafür heranzuziehen, wie politisches Handeln auch in der Klimakrise möglich wäre. Hinzu kommt, dass der dominante Modus der Pandemiebewältigung nicht als emanzipatorisch eingestuft werden kann, wie das folgende Kapitel zeigen wird.

\section{IV. ,Back to normal?' - Der Status quo als Problem}

Die Krisenbewältigung im Kontext der Coronapandemie verbleibt innerhalb einer nationalistischen und kapitalistischen Logik. Somit kann die Coronakrise auch deshalb nicht als Positivbeispiel für den Umgang mit der Klimakrise gewertet werden, weil die Maßnahmen auf eine Rückkehr zum Status quo ausgelegt sind, statt eine notwendige Systemtransformation anzustreben.

\section{Katastrophennationalismus}

Das Regierungshandeln in der Coronakrise folgt einem normativen Nationalismus. So ist die Krisenbewältigung vor allem an nationalen Interessen ausgerichtet, was sich u.a. an den weitgehend ohne vorherige Absprachen erfolgten innereuropäischen Grenzschließungen und an der Konkurrenz um Impfstoffe für die nationale Bevölkerung zeigt. Angesichts einer globalen politischen Ordnung, die vorrangig nationalstaatlich organisiert ist, überrascht diese Form des Umgangs mit der Krise nicht. Im Widerstand 
gegen internationale Zusammenarbeit in der Pandemie schlagen sich Prozesse nieder, die auf bereits vor der Krise existierenden Strukturen beruhen und nun u.a. im Austritt der USA aus der World Health Organisation sichtbar werden. Dabei lässt sich der normative Nationalismus eindeutig nicht als ausschließliche Haltung rechtspopulistischer Regierungen abtun. Auch das Handeln der deutschen Regierung ist an nationalen Interessen orientiert, die teilweise um einen Einbezug der Europäischen Union (EU) ergänzt werden. Dies wird etwa an der geringen finanziellen Unterstützung bei der Pandemiebewältigung für Staaten des Globalen Südens deutlich, gerade im Vergleich zu den innerdeutschen und europäischen Unterstützungsmechanismen. Vernachlässigt wird durch den normativen Nationalismus die Unterstützung für die Bevölkerung von Staaten, die aufgrund globaler Machtverhältnisse über deutlich schlechter ausgestattete Gesundheits- und Sozialsysteme verfügen als beispielsweise Deutschland.

Unbeachtet bleibt auch die Situation Geflüchteter, z.B. in den Lagern an den EU-Außengrenzen, in denen die fatalen hygienischen Bedingungen zur Verbreitung von Covid-19 und anderen Krankheiten beitragen. Grenzen lassen sich dabei als Elemente globaler Herrschaftsstrukturen begreifen, die Individuen filtern, differenzieren und ausschließen. Sie sind die „undemokratische Bedingung, unter welcher sich gegenwärtige Institutionalisierungen der Demokratie herausgebildet haben" (Schwiertz 2019: 647) und konstruieren Zugehörigkeit über Abgrenzung. Da diese Abgrenzung aber imaginiert ist, bleiben Grenzen stets instabil und kontingent (vgl. ebd.: 649f.). Robin Celikates (2020) analysiert die katastrophale Wirkung der Coronapandemie an und durch Grenzen. Grenzschließungen kommunizierten symbolisch an die eigene Bevölkerung, dass der Staat alles unter Kontrolle habe; der politische Charakter der Grenzen gerate so aus dem Blick. Der gegenwärtige Umgang mit Covid-19 sei geprägt von einem „Katastrophennationalismus“ (ebd.), in dem Staaten nur sich selbst retten, statt globale Antworten auf eine globale Krise zu finden.

Anhand dieser Diagnose wird eine Gemeinsamkeit von Corona- und Klimakrise deutlich. Auch in den Verhandlungen zu internationalen Klimaabkommen ist ein Grund für das Scheitern global abgestimmten, solidarischen Handelns die Verfolgung nationaler Interessen. Dabei zeigen sich ähnliche Konfliktlinien wie in der Coronakrise, etwa mit dem Austritt der USA aus dem Klimaabkommen von Paris sowie mit der Forderung von Staaten des Globalen Südens nach stärkeren Emissionssenkungen im Globalen Norden. Auch die Fragen von Flucht, Migration und Grenzen sind hier Thema. In der fehlenden Anerkennung klimabedingter Migration als Fluchtgrund wird ebenfalls eine Form des Katastrophennationalismus sichtbar. 
Auf deskriptiver Ebene blendet der Katastrophennationalismus die tiefgreifenden globalen Abhängigkeiten der Gegenwart aus, weshalb die symbolische Kontrolle durch Grenzschließungen reine Fiktion bleibt. Der globale Kapitalismus ist auf weltweite Lieferketten und Reisewege angewiesen; eine effektive Bewältigung der Coronakrise ist deshalb nur global möglich. Auch im Fall der Klimakrise ist eine nationale Krisenbewältigung absurd, denn Emissionen machen nicht an Grenzen halt und fordern daher grundlegend globale Lösungsstrategien. Auf normativer Ebene ist der Katastrophennationalismus zudem höchst problematisch, denn er reproduziert erstens die gewaltvollen globalen Grenzregime und zweitens die extremen Ungleichheiten in der Betroffenheit von der Klima- und der Coronakrise. Ein emanzipatorisches Vorgehen gegen die Krisen bleibt aus. Die Krisenbewältigung orientiert sich stattdessen an einer Rückkehr zum Status quo vor der Pandemie. Gewaltvolle Grenzregime und globale Ungleichheiten sind aber kein neues Phänomen, sondern waren bereits struktureller Bestandteil der Vor-Corona-Konstellation. Das Gleiche gilt für die Ausrichtung von Politik an kapitalistischen Interessen, die der folgende Abschnitt behandelt.

\section{Stabilisierung oder Systemtransformation}

An dieser Stelle ist es erforderlich, auf das Verhältnis von Ökonomie und Ökologie zurückzukommen. Wie oben ausgeführt, zielen die gegenwärtig getroffenen Maßnahmen zur Pandemiebekämpfung auf die finanzielle Unterstützung, der Wirtschaft ${ }^{\star}$ ab, während ökologische Forderungen und solche nach sozialer und globaler Gerechtigkeit vernachlässigt werden. Zur weiteren Analyse muss die Einbettung dieser Maßnahmen in das kapitalistische System betrachtet werden.

Die ökomarxistischen Autoren John Bellamy Foster et al. (2010) weisen darauf hin, dass der Kapitalismus zur Stabilisierung auf ständige Expansion und auf Wirtschaftswachstum angewiesen ist, was auf einem Planeten mit begrenzten physischen Ressourcen zwangsläufig auf die Zerstörung der ökologischen Grundlagen von Gesellschaft hinauslaufe. Eine Entkopplung wirtschaftlichen Wachstums von ökologischen Ressourcen sei nicht möglich. Mithilfe dieser Analyse lassen sich auch die in der Coronakrise getroffenen Maßnahmen einordnen. An der Dominanz von Ansätzen, die den Konsum erhöhen und ,die Konjunktur ankurbeln` sollen, zeigt sich außer des Natur-Gesellschaft-Dualismus ${ }^{6}$ auch der Wachstumsimperativ kapitalistischer Gesellschaften, der ökologische Zerstörung produziert. 
Das Beispiel der Finanzkrise 2008/09 demonstriert die Folgen eines solchen Handelns: Während die Emissionen während der Krise 2009 um 1,4 \% sanken, stiegen sie im darauffolgenden Jahr um 5,9\% an, wobei Modelle davon ausgehen, dass durch diesen sogenannten Rebound-Effekt die Gesamt-Emissionen sogar höher waren, als sie es ohne die Wirtschaftskrise gewesen wären (vgl. Peters et al. 2012). Zwar ist der geschätzte Einfluss der Coronakrise auf die Gesamt-Treibhausgasemissionen 2020 mit $8 \%$ (Stand Juni 2020) noch deutlich größer als der Einfluss der Finanzkrise (vgl. Evans 2020); in der Bewältigung der aus der Pandemie resultierenden Wirtschaftskrise deutet sich mit den getroffenen Maßnahmen aber ein ähnliches Vorgehen an wie 2009. Ein solches wäre für eine Eingrenzung der Klimakrise fatal, denn um die globale Erderhitzung auf maximal $1,5^{\circ} \mathrm{C}$ zu begrenzen, bräuchte es bis 2030 jährlich einen Rückgang der Treibhausgasemissionen um 7,6\% (vgl. UNEP 2019: 26).

Daran anschließend stellt sich die Frage, was das grundsätzliche Ziel der Krisenbewältigung im Rahmen der Coronapandemie ist. In den aktuell getroffenen Maßnahmen kommt die Dominanz eines Modus zum Ausdruck, der auf eine möglichst rasche und reibungslose Rückkehr zur ,Normalität ${ }^{\star}$ vor der Krise abzielt. Die ökologischen Folgen dieses Status quo sind katastrophal und auch Ungleichheiten und Ausbeutung, die durch die Coronakrise teilweise verschärft wurden, sind keine Folgen der Pandemie, sondern Folgen der sogenannten ,Normalität ${ }^{\star}$ (vgl. dazu Jonas in diesem Band). Sie resultieren u.a. aus sexistischen, rassistischen und kapitalistischen Strukturen, die diese ,Normalität" prägen. Arbeiter"innen in Schlachthäusern lebten bereits vor der Pandemie in ausbeuterischen Arbeitsverhältnissen; Menschen in Pflegeberufen waren auch vor der Pandemie von Überarbeitung und geringer Bezahlung betroffen; Sorgearbeit wurde auch vor der Pandemie überproportional von Frauen* geleistet.

Solche Machtverhältnisse müssen in der Debatte um den Zusammenhang von Corona- und Klimakrise explizit thematisiert werden, um emanzipatorische Kritik am Umgang mit beiden Krisen zu formulieren. Das Konzept Klimagerechtigkeit ist dabei ein wertvoller theoretischer Bezugspunkt. Coventry und Okereke (2017) stellen hier zum einen die massiven Unterschiede bezüglich der Verantwortung für den menschengemachten Klimawandel zwischen Globalem Süden und Norden sowie innerhalb von Gesellschaften heraus. Zum anderen rücken sie die ungleich verteilten Folgen des menschengemachten Klimawandels und der Anpassungsmöglichkeiten daran ins Zentrum der Analyse. Der Ansatz von Klimagerechtigkeit ist dabei an aktivistische Gruppen besonders der Klimabewegung zurückgebunden (vgl. ebd.). Wesentlich ist, dass sich Forderungen nach globaler und sozialer Gerechtigkeit mit solchen nach ökologischer Nachhaltigkeit 
verbinden. Über die Rückbindung an soziale Bewegungen können die Forderungen darüber hinaus als Äußerungen des Politischen analysiert werden, die bestehende Ausschlüsse institutionalisierter Politik problematisieren.

Zusammenfassend verschärfen sowohl Klima- als auch Coronakrise bestehende Unterdrückungsverhältnisse der sogenannten ,Normalität ${ }^{`}$ und müssen daher in Zusammenhang mit diesen analysiert werden, statt ihre Krisenhaftigkeit über die Abweichung vom Status quo zu definieren.

\section{Alle Krisen bekämpfen? - Der Versuch einer Synthese}

Wie deutlich geworden ist, operieren verschiedene gegenwärtige Diskurse über den Zusammenhang von Corona- und Klimakrise einerseits mit Entgegensetzungen und andererseits mit Gleichsetzungen der beiden Krisen. Beides stellt Verkürzungen eines komplexen Wechselspiels bio- und ökosozialer Prozesse dar, wobei besonders die Entgegensetzung beider Krisen einen falschen Dualismus von Natur und Gesellschaft impliziert. Auch eine Gleichsetzung der Corona- mit der Klimakrise ist u.a. aufgrund der verschiedenen räumlichen und zeitlichen Wirkungsweisen vorschnell. In der Coronakrise erscheint eine Rückkehr zu einem Zustand ähnlich jenem vor der Krise deskriptiv eher möglich als in der Klimakrise - auch wenn die tiefgreifenden sozialen, politischen und ökonomischen Folgen der Pandemie sicherlich noch lange fortwirken und zu zurzeit nicht absehbaren gesellschaftlichen Veränderungen führen werden. In der Klimakrise macht zusätzlich aber der grundsätzliche Bruch mit den ökologischen Bedingungen des Holozäns eine Transformation unausweichlich, was Postwachstumstheoretiker*innen treffend als eine Transformation „by design“ oder „by disaster“ benennen (Kallis 2018: 11). Die staatlichen Eingriffe in die Wirtschaftsordnung in der Coronapandemie hätten ein Impuls für eine Transformation sein können. Mit der angestrebten Rückkehr zur ,Normalität ${ }^{\star}$ orientiert sich staatliches wie unternehmerisches Handeln aber am Gegenteil.

Trotz der Unterschiedlichkeit beider Krisen lassen sich aus emanzipatorischer Perspektive aus diesen also in mancher Hinsicht ähnliche Schlüsse ziehen: Corona- und Klimakrise zeigen die Notwendigkeit einer Systemtransformation auf, da sie u.a. nationalistische und kapitalistische Machtverhältnisse verschärfen, die das aktuelle Gesellschaftssystem prägen. Aus einer Perspektive globaler (Klima-)Gerechtigkeit wäre in beiden Krisen global solidarisches Handeln erforderlich. Gefordert ist eine integrative Behandlung multipler sozial-ökologischer Krisen, die die Kritik gesellschaftli- 
cher Unterdrückungsverhältnisse und politischer Ausschlüsse mit Forderungen nach ökologischer Nachhaltigkeit verbindet. Sicherlich kann ein solches Projekt nicht dem Anspruch gerecht werden, alle Krisen zu bekämpfen und wird stets gewisse Ausschlüsse (re)produzieren. Dennoch ist der Versuch einer Systemtransformation und der Überwindung bestehender Ausbeutungsverhältnisse menschlichen und nicht-menschlichen Lebens dringend erforderlich; auch, um überhaupt die ökologischen Rahmenbedingungen für politische Auseinandersetzungen um eine gerechte Ordnung langfristig zu erhalten.

\section{Literaturverzeichnis}

Archer, David. 2009. The long thaw. How humans are changing the next 100,000 years of earth's climate. Princeton, NJ: Princeton Univ. Press.

Celikates, Robin. 2020. Grenzen - Robin Celikates. https://www.youtube.com/watch ? $\mathrm{v}=\mathrm{pWm} 58 \mathrm{feLF} 4 \mathrm{~s} .09 .06 .2020$.

Chakrabarty, Dipesh. 2016. Humanities in the Anthropocene: The Crisis of an Enduring Kantian Fable. New Literary History 47: 377-397.

Clark, Timothy. 2012. Scale. In: Tom Cohen (Hrsg.), Telemorphosis: Theory in the Era of Climate Change 1. Michigan: Open Humanities Press, 148-166.

Coventry, Philip/Okereke, Chukwumerije. 2017. 29 Climate Change and Environmental Justice. In: Ryan Holifield et al. (Hrsg.), The Routledge Handbook of Environmental Justice. London/New York: Routledge Taylor \& Francis Group, 362-373.

Curly Tales. 2020. 6 Things That Prove That The Earth Is Healing / Curly Tales. https:// www.youtube.com/watch?v=R3M908TJG9M. 04.06.2020.

Deutsche Welle News. 2020. Coronavirus: Good for the Environment? / Covid-19 Special. https://www.youtube.com/watch?v=UVxWWW63SdU. 04.06.2020.

Evans, Simon. 2020. Daily global CO2 emissions ,cut to 2006 levels' during height of coronavirus crisis. CarbonBrief. https://www.carbonbrief.org/daily-global-co2emissions-cut-to-2006-levels-during-height-of-coronavirus-crisis.

Foster, John B. et al. 2010. The Ecological Rift. Capitalism's War on the Earth. New York: Monthly Review Press.

Hoppe, Katharina. 2020. Natur - Katharina Hoppe. https://www.youtube.com/watc $\mathrm{h}$ ? $\mathrm{v}=\mathrm{KcWoQ} 8 \mathrm{KdMa} 8$. 11.06.2020.

Horn, Eva/Bergthaller, Hannes. 2019. Anthropozän zur Einfübrung. Hamburg: Junius Verlag.

IPCC (Hrsg.). 2014. Climate change 2014. Impacts, Adaptation, and Vulnerability: Working Group II contribution to the fifth assessment report of the Intergovernmental Panel on Climate Change: Part A: Global and sectoral aspects. Cambridge: Cambridge University Press. 
Kallis, Giorgos. 2018. Degrowth. Newcastle upon Tyne: Agenda Publishing.

Latour, Bruno. 2017. Facing Gaia. Eight Lectures on the New Climatic Regime. Cambridge, UK/Malden, MA: Polity.

Lehmann, Armin. 2020. FDP warnt vor Verschuldungsrisiko durch Konjunkturpaket. Der Tagesspiegel. https://www.tagesspiegel.de/politik/morgenlage-aus-der-hau ptstadt-fdp-warnt-vor-verschuldungsrisiko-durch-konjunkturpaket/25930274.ht $\mathrm{ml}$.

Moore, Jason W. 2017. The Capitalocene, Part I: on the nature and origins of our ecological crisis. The Journal of Peasant Studies 44: 594-630.

Peters, Glen P. et al. 2012. Rapid growth in CO2 emissions after the 2008-2009 global financial crisis. Nature Climate Change 2: 2-4.

Schwiertz, Helge. 2019. Grenzen. In: Dagmar Comtesse et al. (Hrsg.), Radikale Demokratietheorie. Ein Handbuch. Berlin: Suhrkamp, 647-657.

Tsing, Anna L. 2019. On Nonscalability. Common Knowledge 25: 143-162.

UNEP. 2019. Emissions Gap Report 2019. Nairobi. 